\title{
Red DESC-ARUANDA: 10 años de apoyo al cumplimiento de los derechos para la reconstrucción del buen vivir de los pueblos autóctonos en América Latina
}

\author{
DESC-ARUANDA Network: 10 Years of Supporting the \\ Fulfillment of Rights for the Reconstruction of Good Living of \\ Indigenous Peoples in Latin America
}

\author{
Deborah Leal-Rodrigues \\ Universidad Nacional \\ Heredia, Costa Rica \\ dlealrodrigues@yahoo.com.br \\ Heidy Vega-García \\ Universidad Nacional \\ Heredia, Costa Rica \\ heidy.vega.garcia@una.cr
}

Recibido: 09/23/2014 Aceptado: 06/29/2016

Resumen. Cambios contemporáneos en el contexto de vida de las comunidades autóctonas afectan directamente el cumplimiento de sus derechos económicos, sociales y culturales (DESC). La intensificación de la violencia local; la falta de alternativas de sobrevivencia, y la presión histórica por tierras y recursos, agudizadas por la inoperancia de sistemas de seguridad social, salud y educación estatales, dieron paso a la conformación de la RED DESC-ARUANDA, en 2004, como un espacio de autoformación, dirigido a favorecer el buen vivir comunitario, mediante el fortalecimiento de la voz de los pueblos y el diálogo intercultural. Desde 2008, la plataforma DESC ARUANDA/ CEG/UNA es parte del proceso de trabajo humanista del CEG/UNA, y realiza investigaciones estudiantiles, para consolidar el poder de resistencia local, fortaleciendo sus movimientos sociales, que claman por la conservación de la diversidad cultural, y ecológica de sus tierras y territorios. En el contexto histórico latinoamericano, es posible considerar que la mayor conquista de los movimientos sociales sea la consolidación de redes que incorporan el poder biopolítico de la multitud (Negrini, 2004); generan cambios en el imaginario local y; abren 
Revista Universidad en Diálogo • Vol. 6, N. ${ }^{\circ} 2$, Julio-diciembre 2016, pp. 75-92

ISSN 2215-2849 • EISSN: 2215-4752

DOI: http://dx.doi.org/10.15359/udre.6-2.5

canales de continuidad sostenible hacia la reconstrucción de futuros autodeterminados, en relaciones interculturales para la reconstrucción de la vida como un todo.

Palabras clave: pueblos autóctonos, educación intercultural, sostenibilidad.

Resumo: Mudanças contemporâneas no contexto de vida das comunidades autóctones afetam diretamente o cumprimento dos seus direitos econômicos, sociais e culturais (DESC). A intensificação da violência local; a falta de alternativas de sobrevivência, e a pressão histórica por terras e recursos, complicadas pela inoperância dos sistemas de segurança social, saúde e educação pública, deram passo à conformação da REDE DESC-ARUANDA, em 2004, como um espaço de auto-formação, dirigido $\grave{a}$ favorecer o "bom viver" comunitário, pelo fortalecimento da voz dos povos e o diálogo intercultural. Desde 2008, a plataforma DESC ARUANDA/CEG/UNA é parte do processo de trabalho humanista do CEG/UNA, e realiza pesquisas estudantis, para consolidar o poder de resistência local, fortalecendo os movimentos sociais, que clamam pela conservação da diversidade cultural e ecológica, das suas terras e territórios. Dentro do contexto histórico latino americano, é possível considerar que a maior conquista dos movimentos sociais seja a consolidação de redes que incorporam o poder biopolítico da multidão (Negrini, 2004); provocam mudanças no imaginário local e; abrem canais de continuidade sustentável à reconstrução de futuros autodeterminados, em relações interculturais para a reconstrução da vida como um todo.

Palavras chave: povos autóctones, educação intercultural, sustentabilidade.

Abstract. Contemporary changes in the context of the indigenous communities' life directly affect the fulfillment of its economic, social and cultural rights (ESCR). The intensification of local violence, the lack of alternatives for survival, and the historical pressure for land and resources, coupled with the inadequacy of social security systems and state health and education, gave way to the conformation of the ESCR-NET-ARUANDA, in 2004, as a space of self-education, aimed at favoring the community's good living, through the strengthening of the peoples' voice and the intercultural dialog. Since 2008, the DESC ARUANDA/CEG/A platform is part of the humanist working process of the CEG/A, and it conducts student investigations to consolidate the power of local resistance, strengthening its social movements which are clamoring for the preservation of cultural and ecological diversity of their lands and territories. In the historical Latin America context, it is possible to consider that the greatest conquest of the social movements is the consolidation of networks that feature the biopolitical power of the multitude (Negrini, 2004), generate changes in the local imaginary, and open channels of sustainable continuity toward the reconstruction of future self-determined individuals, in intercultural relations for the reconstruction of life as a whole.

Keywords: indigenous peoples, intercultural education, sustainability. 


\section{Antecedentes: Factores considerados en el proceso de constitución de la red DES-ARUANDA}

Existen contradicciones entre reconstituir personas y educación -entendida en el marco de la reconstrucción de la sostenibilidad como continuidad posible, digna y autodeterminada- y las eternas necesidad/dependencias constituidas de los recursos externos. ¿Cómo pensar en una reforma profunda de la educación pública sin la reconstrucción del ser? Se considera que la educación debería abordar el-desarrollo-de-la-vida-misma, el proceso de reconstitución del mundo, de su propia ontología. De este modo, hay que reconocer la importancia de la sostenibilidad de la propia producción o reproducción social y cultural. ¿Cuáles, entonces, deben ser las consideraciones mínimas en el proceso de reconstrucción de una educación intercultural, que permitan la sobrevivencia de lo propio? ¿Cómo vencer las inevitables barreras del lenguaje, de las condiciones innombrables de comunicación con los espacios de decisión y los espacios económicos? De la distancia entre lo que debería ser un plan para el buen vivir y la sostenibilidad de la vida local, la reconstrucción de las posibilidades de la vida local depende, en este caso, de la reconstrucción de los saberes locales, de la sistematización y la complementariedad, con elementos o saberes de otras ciencias y, así, la recuperación del equilibrio, del hilo de la vida sostenible en comunidad.

La Universidad Nacional (UNA) históricamente ha actuado en comunidades indígenas, lo que ha resultado en una popularidad que provoca una expectativa latente en lo local. Entre los años 2008-2013, el proyecto Plataforma de los DESC-ARUANDA llevó a cabo una serie de actividades estudiantiles en los territorios indígenas de Costa Rica: se realizaron diagnósticos comunitarios participativos, en coordinación con el estudiantado, y actores y actoras locales. Los resultados han sido sistematizados y divulgados a nivel comunitario (internet, boletines y otros) (ver Anexos).

La Red de Derechos Económicos, Sociales y Culturales -Aruanda (RED DESC-ARUANDA) actualmente cuenta con colaboradores de las comunidades autóctonas; líderes, mujeres y hombres, jóvenes y adultos mayores, maestros y organizaciones indígenas, a nivel nacional e internacional. El enlace se ha mantenido de manera virtual y con el trabajo de campo estudiantil en comunidades, realizado en el marco de las actividades de cursos regulares del CEG (Centro de Estudios Generales), estudiantes y personal del CEG, así como con el aporte ad honoren de nuestros colaboradores a nivel internacional, especialistas y pueblos indígenas. 
Revista Universidad en Diálogo • Vol. 6, N. ํㄹ 2, Julio-diciembre 2016, pp. 75-92

ISSN 2215-2849 • EISSN: 2215-4752

DOI: http://dx.doi.org/10.15359/udre.6-2.5

Como meta a largo plazo este proyecto avizora contribuir para la estructuración de un espacio académico en el CEG, el cual funcione como: 1) Oficina virtual de contacto entre la comunidad universitaria y los pueblos indígenas para la reconstrucción de su buen vivir comunitario; 2) Acervo de datos permanente sobre pueblos Indígenas y DESC; 3) Espacio de enlace entre pueblos indígenas y comunidad universitaria; 4) Espacio de apoyo a los estudiantes indígenas que ingresan al CEG y; 5) Observatorio permanente para el cumplimiento de los DESC de los pueblos indígenas en Costa Rica.

Entre nuestras estrategias de empoderamiento e incidencia de los pueblos indígenas en el escenario nacional e internacional se ha buscado facilitar el diálogo de saberes a nivel internacional de los actores indígenas nacionales, en el marco del cumplimiento de los DESC y continuidad sostenible de la vida en sus territorios.

Este artículo delinea las motivaciones y el proceso constitutivo de la red DESC-ARUANDA, y sus 10 años de trayectoria en el trabajo con las comunidades autóctonas. Nace en el periodo de final de siglo XX, en la contraposición aguda de las luchas y conquistas de los movimientos sociales en espacios amazónicos, y la búsqueda de formas de desarrollo alternativo afectadas por los intereses y estrategias nacionales de desarrollo, que continúan fundamentadas en las riquezas de los territorios ancestrales, en el marco de la emergencia de los nuevos gobiernos de izquierda, en América Latina.

\section{Contexto político-geográfico de la región}

\section{Contexto de emergencia de la RED DESC ARUANDA en Brasil}

Amazonía es tierra de piratas que se reciclan en el tiempo cambiando sus botines con cada nuevo descubrimiento en la biodiversidad local. El boom del caucho (Hevea brasiliensis) trajo riquezas únicas para las contadas ciudades de la región (Belém, Manaus, Iquitos y Lima). En el siglo XX, la caída en el precio del caucho ${ }^{1}$ fue compensada por la extracción de la

Uno de los primeros eventos comprobados de biopiratería, pues fue llevado por plantadores ingleses hasta Asia, donde se reprodujo bien sin competencia. A finales del siglo XIX Malasia ya estaría produciendo las primeras pelotas de caucho. Las oligarquías locales cayeron. Los pueblos indígenas se tranquilizaron (Bush y Sachs, 1981). 
castanha-do-pará (nuez del Brasil), drogas del sertão², fibra de yute, entre otras. La economía amazónica floreció con la esclavización y el exterminio de indígenas "rebeldes" y de las poblaciones mestizas que se formaban a lo largo de cada río.

La destrucción se intensificó en el siglo XX con la introducción de monocultivos y la ganadería, apertura de carreteras y la entrada de colonos sin tierra y extractores de productos forestales. Las nuevas formas de producción agropecuaria fracasaron y sirvieron para justificar que las únicas potencialidades de la región serían la minería, la extracción de maderera, caucho, petróleo, gas y del potencial energético; actividades que generaron importantes ingresos a los estados, en gran parte utilizados para el pago de la deuda y que, además, se diluyeron en las concesiones injustas entre gobiernos, que en diferentes casos han favorecido a las compañías transnacionales. Recientemente, la posibilidad de pago por los servicios ambientales también genera expectativas, esta es rechazada por los movimientos sociales locales.

Los pueblos amazónicos resisten y tratan de reconstruir sus territorios cada día, día con día. Parte de esta lucha ha rendido la creación de normativas internacionales y nacionales, que no siempre logran favorecer la autodeterminación de las colectividades, pero que se transforman en insumos para la resistencia y reconstrucción de la autodeterminación. Según el texto de la Constitución de 1988, un año después de la ratificación del Convenio 169 de la OIT (1987), los indígenas de Brasil habrían conquistado el derecho a su autodeterminación; sin embargo, normativas secundarias reducen ampliamente el espectro real de indígenas que pueden recuperar el derecho sobre su tutela, estos fueron únicamente los llamados indios urbanos, o los que tienen segundo grado escolar completo, hablan portugués, entre otros detalles. Mientras tanto, los territorios indígenas continúan sin ser titulados, o titulados a nombre del gobierno federal o de los gobiernos estaduales y municipales: así, realmente nada es de los indígenas, ni siquiera el derecho de mandar en sí mismos.

Los indígenas no tienen otra alternativa, más que depender de la FUNAI para lograr la titulación de sus tierras. Oficialmente, la Unión continúa siendo la responsable legal por los indígenas, estando dentro de sus atribuciones la real propiedad de las tierras indígenas y de sus recursos natu-

Diferentes plantas medicinales y aromáticas recolectadas artesanalmente y exportadas desde el período colonial, como canela, salsaparrilla, marapuama, copaíba, cascara sagrada. También se exportaban pieles de animales, animales de compania como loros, monos, pájaros, entre otros. Animales como el manatí (Trichechus spp.) o la tortuga de agua dulce (Charapa sp.), así como las nutrias (Mustelidae) (Bush y Sachs, 1981). 
rales, así como la administración de la vida indígena, incluyendo el derecho a la reproducción de sus prácticas culturales, seguridad social, salud y educación. Atribuciones aseguradas en el párrafo XI, del artículo 20 de la Constitución brasileña de 1988 y en las diferentes leyes, decretos, portarías que finalmente definen la FUNAI como la tutora de los indígenas y la Fundação Nacional de Saúde (FUNASA) como la ejecutora de una salud diferenciada para estas poblaciones. La educación bilingüe e intercultural es una atribución del Ministerio de Justicia y de Educación ${ }^{3}$. En la última década, el estado de Pará, como otros estados nacionales, viene financiando y sustentando parte de las acciones sociales en comunidades indígenas.

Al observar el citado caso de los DESC de las culturas amazónicas, se determinó que de los procesos de consolidación de las Repúblicas hacia el desarrollo de los Estado-Nación latinoamericanos y sus proyectos integracionistas se pasó al abandono y a la actual tentativa de dar asistencia a las minorías étnicas; tentativa que no es más que reacción a las presiones internas y externa por un nuevo tratamiento a las colectividades y minorías étnicas. La postura desde los Estados, por lo general, ha sido de incluir

\footnotetext{
Assim como cabe ao Congresso nacional legislar sobre as populações indígenas (Art. 22, p. XIV), as atividades de caça, pesca, florestas, de conservação da natureza e recursos naturais (Art. 24, p. VI), e autorizar em terras indígenas a exploração e o aproveitamento de recursos hídricos e a pesquisa e lavra de riquezas minerais. No Art. 215 garante o pleno exercício dos direitos culturais das minorias étnicas, definidas como culturas populares, indígenas e afro-brasileiras. Por outro lado, o Art. 231 reconhece os direitos dos indígenas a sua organização social, costumes, línguas, crenças e tradições, e os direitos originários sobre as terras que tradicionalmente ocupam, competindo à União demarcar, proteger e fazer respeitar todos os seus bens. Sendo esta, o reflexo da adesão a Convenção 169 da OIT, que, no entanto não se reflete na tutela dos indígenas, nos direitos assegurados nos primeiros capítulos da própria Constituição e da Lei No. 10.683, de 28 de maio de 2003. E no Estatuto da FUNAI, de 1967, confirmado pela portaria No. 542 de 1993 -contrariando o 169OIT-, que ratifica o exercício da tutela dos indígenas e das suas comunidades, a posse inalienável das terras, o resguardo da identidade diferenciada do indígena no contexto da sociedade nacional, assim como gerir o patrimônio indígena, as pesquisas nos seus territórios e promover uma educação de base e saúde apropriada. Ao mesmo tempo, que contraditoriamente garante o direito ao domínio dos códigos da sociedade nacional, a participação na vida nacional em igualdade de condições. Assim como o direito à auto-sustentação segundo as suas especificidades culturas, ambientais, tecnológicas e sócio-econômicas. E o direito a uma saúde diferenciada ratificado no Decreto No. 3156 de 1999, que dispõe a promoção, proteção e recuperação da saúde e do equilíbrio bio-psico-social, complementado pelas práticas da medicina indígena. Isto buscando a redução da mortalidade materna e infantil, a interrupção do ciclo de doenças transmissíveis, o combate a desnutrição e ao deterioro ambiental, sendo assegurando no SUS e executado pela FUNASA, direitos ratificados na portaria No. 254 de 2002, que entre outras diretrizes, garante a preparação dos recursos humanos para a atuação em contexto intercultural, o uso adequado de medicamentos, um ambiente saudável e o controle social. O abastecimento de água é regulamentado na Portaria da FUNASA, No. 479, de 2001. Os direitos a educação intercultural das comunidades indígenas está assegurado no Art. 79 da Lei No. 9394 de 1996 (Ver "Legislação Indigenista Brasileira” CGDOC/FUNAI).
} 
estos contingentes en sus agendas y de buscar alternativas, en procesos que caminan lentamente, debido a tres motivos básicos: falta de recursos humanos y económicos; falta de conocimientos e instrumentos apropiados para tratar con las singularidades de las culturas y colectividades; y porque las demandas de las colectividades son imposibles de cumplir.

Considerando los macro objetivos de los gobiernos y sus invariables planes de atender a toda una nación con los recursos naturales generados a partir de la explotación de las tierras y territorios de las minorías étnicas; teniendo en cuenta que, casi nunca, reciben beneficios directos y justos ante las múltiples invasiones de que son víctimas, muchas de las luchas son infructuosas -sin solución-, pues los objetivos de los poderes económicos, si se cumplen, llevaran al fin de uno de los grupos.

\section{Los movimientos sociales, sus motivaciones, prioridades y estrategias}

Los movimientos sociales son orgánicamente constituidos con la acción organizada de hombres y mujeres, lo que implica la movilización y autoeducación de las colectividades. Desde sus nuevas manifestaciones, reivindican la vida plena, el respeto por todas las formas de vida y son, así, tal vez, el cambio del individuo máquina, esclavo del sistema, hacia la autodeterminación de colectividades que pasan a integrarse en procesos más amplios que desafían el poder y demuestran que el futuro ya llegó.

A partir del Convenio 169 de la OIT, los pueblos indígenas y tradicionales recuperaron, en el papel, el derecho a la autodeterminación y, con esto, el derecho al manejo integral de sus territorios colectivos. La normativa ha sido ratificada e incorporada en las constituciones de gran parte de los países amazónicos. Al mismo tiempo, los recursos naturales de los territorios ancestrales se mantienen como una utopía ante los macrointereses de los estados y naciones, los cuales se reflejan en determinaciones que se sobreponen a las formas de vida de las poblaciones locales.

\section{Interculturalidad y reconstrucción cultural}

En el proceso de dominación cultural, la comunicación (educación que comunica) no solo expresa, sino también organiza el movimiento de la vida local y su relación con lo global. Organiza el movimiento, multiplicando y estructurando interconexiones a través de redes. Expresa el movimiento y controla el sentido y la dirección del imaginario local, que se transmite a lo 
Revista Universidad en Diálogo • Vol. 6, N. ํㄹ 2, Julio-diciembre 2016, pp. 75-92

ISSN 2215-2849 • EISSN: 2215-4752

DOI: http://dx.doi.org/10.15359/udre.6-2.5

largo de estas conexiones comunicativas. En otras palabras, guía y canaliza el imaginario dentro de la máquina comunicativa. El lenguaje comunica, crea nuevas subjetividades, las relaciona entre sí y las ordena nuevamente. Las máquinas de comunicación se imponen o pasan a integrarse al imaginario y simbólico de las culturas locales, dentro de su propia trama, de su espacio biopolítico local.

Se puntúan tres momentos básicos, definidos por los actores indígenas en diferentes países:

- Interculturalidad para la resistencia: se caracteriza por ciertas formas y espacios de relación muy limitados entre culturas. No existe la perspectiva de construcción de la equidistancia, sino de la explotación, asimilación y exterminio. No se trabajan las contradicciones de las culturas.

- Interculturalidad para la negociación: se caracteriza por el desarrollo de ciertas habilidades políticas de los dirigentes indígenas, para ocupar ciertos espacios de representación u cuotas de poder. En estos, tratarán de hacer valer los derechos de su cultura y de sus comunidades. En esta etapa, las relaciones se basan en la tolerancia.

- Interculturalidad para la reconstrucción: solo es posible mediante el desarrollo afirmativo de la equidistancia entre culturas. Se da el espacio suficiente para que las culturas minoritarias puedan reafirmar su cosmovisión e intereses genuinos de vida, $\mathrm{y}$ desplegar su propia visión de una continuidad sostenible. En esta etapa, las relaciones se basan en el respeto mutuo y profundo a la diversidad humana y ambiental.

Considerando el que educa, es fundamental tener en cuenta la importancia y límites del bilingüismo y de los conocimientos que quien observa desde lo externo podrá tener de la cosmovisión de las culturas. Es primordial considerar que el problema de la conformación de un proceso apropiado de desarrollo de la educación intercultural transciende el tema de la lengua. Este se extiende a la comprensión y la vivencia de las prácticas cotidianas para la reproducción de la vida, a las formas y redes de comunicación y a los conocimientos sagrados de una cultura. En esto, es importante definir precisamente qué conocimientos son de interés de la comunidad. Por ejemplo, qué tipo de matemáticas quieren recibir; o qué lenguas deben ser priorizadas. 
Los pueblos indígenas luchan de diferentes formas y perspectivas para conservar su identidad; y esta se encuentra en la vida como un todo. Un sistema educativo que considera una reforma de la educación para la sostenibilidad de la población local debe ser pensado considerando su posibilidad de reproducción material digna. Debe considerar la reconstrucción de identidades y, desde ahí, de ciudadanías locales, que saben vivir sin destruir el ambiente.

Desde esta perspectiva, las culturas indígenas tienen destrezas constituidas en el diario convivir con el entorno local, y sus tecnologías y sistemas adaptativos, realísticamente, más aptos para sobrevivir con sus estrategias y técnicas ancestrales. El papel de universidad pública, como agente enculturador, debe ser el de fortalecer la cultura, sus conocimientos y prácticas. Si se busca la no asimilación forzada de las culturas y pueblos indígenas, se debe pensar en manejar mejor el tiempo de enculturación (horas clase) y adaptar el currículo a las estéticas y tiempos culturales.

Con esto, resulta indispensable incluir a la familia, de manera respetuosa al proceso educativo. Es importante, antes de sugerir cambios, por ejemplo, observar mejor a las familias, ofrecer ayuda estratégica, considerando sus problemas y necesidades; pero respetando sus formas de reproducción cultural ancestral. Es trascendental recordar que las identidades indígenas (liderazgos) emergen como identidades para la resistencia-identidades creadas y recreadas bajo relaciones desiguales de poder-.

Del mismo modo, se contrapone la estética, como expresión/observación de la vida real y sus elementos (lectura y reflexión), a la lógica, que corresponde a asociaciones necesarias de ideas, previsión, previsibilidad, que nunca cambia. Las instituciones indígenas pueden, aunque también ellas se ven "naturalmente" arrastrando un flujo de temporalidad, mantenerse a distancia constante de la contingencia histórica y de la inmutabilidad de un clan.

Se hace necesaria una reforma de la educación desde abajo, en la reconceptualización de estéticas que responden a planes de desarrollo apropiados para las personas de las diferentes culturas, considerando un perfil de egreso, que se inscribe en un perfil de ciudadano consciente de las fuerzas de asimilación e integración impuestas, al mismo tiempo, del deber impuesto de contribuir a un sistema, a un Estado-Nación, con atributos superiores al de sus propias nacionalidades o identidades tribales, así como la necesidad impuesta de pasar a contribuir a sus proyectos macro-económicos nacionales y superpuestos por los proyectos transnacionales. 
Revista Universidad en Diálogo • Vol. 6, N. ํㄹ 2, Julio-diciembre 2016, pp. 75-92

ISSN 2215-2849 • EISSN: 2215-4752

DOI: http://dx.doi.org/10.15359/udre.6-2.5

¿Cuáles, entonces, deben ser las consideraciones mínimas en el proceso de reconstrucción de una educación intercultural, que permitan la sobrevivencia de lo propio? ¿Cómo vencer las inevitables barreras del lenguaje, de las condiciones innombrables de comunicación con los espacios de decisión y los espacios económicos? De la distancia entre lo que debería ser un plan para el buen vivir y la sostenibilidad de la vida local, considerando esta como vida biológica; pero también, como vida social y cultural, con contextos económicos propios.

Se necesita estructurar una buena comunicación física entre mundos, pero estratégicas para la sobrevivencia cultural. Por esto, se necesita reconstruir prioritariamente lo local: como los sistemas de producción local y la soberanía alimentaria; la capacidad de desarrollar y reproducir sistemas y especies de cultivos propios y autóctonos; las estrategias de limpieza y reciclaje propias; reconstrucción de prácticas culturales; restablecer el equilibrio entre salud y enfermedad, mediante el desarrollo de un sistema de salud integral, que relaciona ambos conocimientos como fortalezas específicas y, a la vez complementarias, de mantenimiento de la población con calidad de vida, desde los saberes locales.

La reconstrucción de las posibilidades de la vida local depende, en este caso, de la reconstrucción de los saberes locales, de la sistematización y la complementariedad, con elementos o saberes de otras ciencias; y así, la recuperación del equilibrio, del hilo de la vida sostenible en comunidad. Que pasa a estar estratégicamente comunicada con los elementos que son positivos, desde sus necesidades de resistencia reconstrucción, a cada día.

\section{De la teoría a la práctica: El establecimiento de la plataforma DESC ARUANDA en la UNA-COSTA RICA/2008}

\section{Investigación-acción humanista: fases y desarrollo de estrategias de trabajo}

Desde el año 2008, se han realizado diferentes estudios comunitarios transversales e investigación-acción participativa, para el cumplimiento de los DESC de las comunidades, familias y ciudadanos y ciudadanas de Costa Rica. Entre los años 2008-2012, se realizó un diagnóstico del contexto DESC de los pueblos y territorios indígenas de Costa Rica; el cual se encuentra sistematizado en el Informe ARUANDA DESC - Capítulo Costa Rica/2012 y en la base de datos DESC-ARUANDA. 
Entre los principales resultados de este período, se determinó un panorama de aislamiento relativo en las diferentes comunidades, y de resistencia local no organizada a la interminable invasión de sus territorios y expansión de comercios y fincas de no indígenas; la búsqueda propia de alternativas de sobrevivencia afuera de sus territorios ancestrales y la confirmación de que un $50 \%$ de los indígenas de Costa Rica hoy viven, aunque temporalmente, en las principales ciudades del país.

La inexistencia de políticas estatales, sistemáticas y articuladoras de la complejidad de las realidades y necesidades locales es, a la vez, causa y síntoma de la fragilidad de la resistencia organizada indígena a nivel local. En otras palabras, las fuerzas vivas locales no tienen poder de decisión sobre la reconstrucción de sus realidades y la preservación de su matrimonio material e inmaterial.

En los diferentes contextos explorados, se pueden notar diversos grados de aislamiento y segregación de la población local. De manera que la lucha por sus derechos solo se hace en la base de la sobrevivencia individual, familiar y clánica o asociativa, como organizaciones productivas, como máximo. A nivel comunitario, los maestros del sistema nacional de educación pasan a ser los líderes con poder local. Son ellos, por ejemplo, los que ejecutan el censo y los únicos con real y sistemática entrada dentro de los territorios.

Al fin, pareciera que el futuro de las comunidades reposa en los brazos espléndidos de la obra educadora nacional, que se encarga de la transculturación e integración forzada de los contingentes indígenas al imaginario identitario de Costa Rica, aunque no asegura su ingreso concreto en los escenarios económicos y laborales del país. Enuncia una realidad en la cual nueve de cada diez estudiantes indígenas no terminan el colegio en Costa Rica, carecen de alternativas laborales dentro de su comunidad y son incluidos en los peores espacios de venta de mano de obra del país, como las plantaciones bananeras.

\section{El portal virtual y enlace comunitario}

Entre las conclusiones principales del Informe DESC-ARUANDA/2012, se encontró que los pueblos indígenas en Costa Rica buscan un espacio de inclusión que permita que reproduzcan sus culturas y tengan una calidad de vida relativamente coherente con el patrón nacional. Es importante favorecer acciones concretas que apoyen el trabajo colectivo y que considere la historia de exterminio de que fueron víctimas, además, que reconozca que esta historia no se ha concluido ni borrado de su memoria colectiva. 
Revista Universidad en Diálogo • Vol. 6, N. ํㄹ 2, Julio-diciembre 2016, pp. 75-92

ISSN 2215-2849 • EISSN: 2215-4752

DOI: http://dx.doi.org/10.15359/udre.6-2.5

Es fundamental conocer cómo se dio el proceso de conquista y desarticulación de la vida indígena; y el proceso de reconstitución vivido por las culturas sobrevivientes hasta el presente siglo, considerando su grado de distanciamiento, físico y cultural, de la sociedad; el grado de intervención y apropiación de sus recursos naturales; los procesos político-organizativos y el imaginario cultural de cada lugar; así como la ampliación de la comprensión de las relaciones interculturales e históricas entre los pueblos, para proponer mejores formas de trabajo colectivo, que provoquen el desarrollo de alianzas estratégicas, favorecedoras de la auto-determinación y el futuro de todos.

Se considera que la persistencia de los elementos, saberes y prácticas culturales ancestrales en América Latina se dio y se viene dando, por la misma necesidad de sobrevivencia, aun cuando las políticas integracionistas apunten al exterminio de la vida cultural e incluso biológica de las poblaciones autóctonas, las cuales han incluido, e incluyen, en diferentes países, estrategias, más o menos estructuradas, como:

1. Usurpación de los territorios ancestrales y compresión de las áreas de uso cultural, hasta llegar al extremo de las reservas indígenas.

2. Separación de familias, clanes y comunidades.

3. Eliminación de los elementos y formas propias de reproducción de la vida, como la caza, pesca, agricultura, biodiversidad y ritualidad.

4. Eliminación abrupta de las lenguas locales y de los momentos y formas de reproducción del "bagaje" cultural en los mitos y actividades colectivas.

5. Cuanto más rápido posible, el establecimiento de un sistema de enseñanza normal y religioso, que los aísla completamente de los escenarios de reproducción de los conocimientos culturales.

Actualmente, el rescate de las cosmovisiones ancestrales comprende la reunión de los fragmentos visibles dentro de las culturas indígenas contemporáneas, a partir de la reconstrucción de los elementos y marcos más amplios de las culturas; de su historia de exterminio y sobrevivencia; de los testimonios de las personas de conocimiento; de la recopilación de sus memorias, mitos, historias, lenguas y prácticas cotidianas de sobrevivencia. 


\section{En el momento, ARUANDA desarrolla un modelo de trabajo comunitario en coordinación con las actividades académicas y los cursos impartidos en el CEG.}

Los cursos son de carácter teórico-práctico. Se presentan los logros, dificultades y las necesidades de trabajo comunitario; y los estudiantes desarrollan un proyecto de investigación-acción, justificado. El curso empieza con un foro metodológico virtual y un debate teórico presencial, considerando conocimientos pertinentes en: 1) Cosmovisiones y medicina alternativa. 2) Pensamiento y sociedad. 3) Ambiente y producción. 4) Trabajo social.

En el foro metodológico virtual, los estudiantes conocen los temas relacionados a: 1) Las comunidades locales. 2) Su problemática. 3) Los temas DESC. 4) Sus necesidades. 5) Las posibles líneas y metodologías de trabajo de campo. En el debate teórico presencial los estudiantes conocen los temas relacionados a: 1) El origen de la humanidad. 2) Evidencias materiales de las culturas antiguas. 3) Pensamiento. 4) Espiritualidad. 5) Sobrevivencia cultural.

En el foro metodológico virtual elaboran un proyecto de investigación, que consta de una exploración teórica y una incursión a una comunidad local o virtual. Para esta realización, los estudiantes se dividen en cuatro grupos de trabajo: 1) cosmovisión; 2) pensamiento; 3) ambiente; 4) sociedad. En cada semestre, avanzan en algunas comunidades nuevas y exploran nuevos temas, produciendo resultados que serán añadidos a la base de datos DESC-ARUANDA, según su especificidad, para futuras reediciones, a ser devueltas a las comunidades y grupos visitados.

El proyecto tiene como meta, a largo plazo, generar información pertinente para la preservación de la memoria cultural de los pueblos autóctonos del planeta e incentivar nuevos programas de estudios humanísticos, enfocados en el buen vivir, los DESC y la reconstrucción cultural y de la vida como un todo. El enfoque de acción, investigación y reconstrucción practicado produjo resultados de investigación y premisas para el abordaje de las comunidades, en la cooperación para su buen vivir y el cumplimiento de sus DESC. Estos resultados, reunidos, conforman una trayectoria de trabajo que avizora su continuidad, en el establecimiento de programas más sistemáticos de investigación, divulgación de información y contribución desde los espacios académicos para el proceso de autodeterminación. 
Revista Universidad en Diálogo • Vol. 6, N. ํㄹ 2, Julio-diciembre 2016, pp. 75-92

ISSN 2215-2849 • EISSN: 2215-4752

DOI: http://dx.doi.org/10.15359/udre.6-2.5

\section{El futuro que se avizora: El plan de formación en estudios humanísticos, interculturalidad y DESC del Centro de Estudios Generales}

El plan de formación en estudios humanísticos, interculturalidad y DESC, busca enlazar los intereses de la Universidad Nacional con el desarrollo de alternativas, estrategias y contenidos temáticos pedagógicos interculturales, apropiados para los pueblos indígenas de Costa Rica, en el marco del cumplimiento de sus derechos económicos, sociales y culturales (DESC). Propone el rescate de las experiencias acumuladas por el Proyecto plataforma de derechos económicos, sociales y culturales (DESC) de los pueblos indígenas - ARUANDA/CEG. Se pretende fortalecer la comprensión de actores claves acerca del contexto histórico vivido por sus territorios y el proceso de reconstitución cultural. Se aborda la problemática local, desde la agenda indígena de los pueblos y comunidades indígenas de Costa Rica, con la meta de favorecer la reconstrucción del buen vivir comunitario y el cumplimiento de sus DESC.

El plan de formación en estudios humanísticos, interculturalidad y DESC de los pueblos indígenas busca realizar un aporte a la educación pública de Costa Rica, la cual garantice los derechos humanos de los pueblos indígenas asegurados en la actual Constitución y Ley de Educación No. 7316, del 03 de noviembre de 1992 y ratificados por Costa Rica en el Convenio 169 de la OIT sobre Pueblos Indígenas y Tribales en Países Independientes, publicado en el Diario Oficial La Gaceta No. 234, del 04 de diciembre de 1992.

La propuesta se enlaza a los objetivos del Plan estratégico del Centro de Estudios Generales en lo que dice respeto al compromiso de: 1) Promover la construcción de conocimiento, valores y aptitudes desde una visión humanística para empoderar a los docentes, administrativos y estudiantes con un nuevo paradigma en la enseñanza de las humanidades. 2) Propiciar la reflexión, la discusión y la producción de conocimiento acerca de la diversidad cultural, el patrimonio histórico y natural de la realidad nacional, latinoamericana y universal, para la construcción continua de una cultura de paz. 3) Brindar a la comunidad espacios y oportunidades de educación continua, por medio de los distintos programas, proyectos y actividades.

Además, se propone realizar un aporte para la autoeducación comunitaria indígena, mediante el fortalecimiento de un espacio virtual, red DESC-ARUANDA y la elaboración de insumos estratégicos para el proceso autoeducativo comunitario. Se espera innovar la oferta de cursos y la pedagogía del CEG, con una contribución para la formación de las comunidades indígenas de Costa Rica en estudios humanísticos, interculturales y 
DESC, considerando del compromiso del CEG de contribuir en la construcción de un nuevo humanismo, mediante la generación de una oportunidad de formación digna de los actores y actoras indígenas, en la perspectiva de la construcción de nuevas ciudadanías del buen vivir en el siglo XXI.

Es importante crear espacios de comunicación en los cuales los pueblos indígenas tengan oportunidad de reproducir sus propias formas de desarrollo: entendidas hoy por los movimientos indígenas como continuidad sostenible o buen vivir, como forma propia de encontrar un proceso de sobrevivencia en armonía con sus culturas ancestrales, visión de futuro y reconstrucción.

\section{Conclusiones y futuras acciones propuestas de la red RESC ARUANDA}

Entre las necesidades básicas de los pueblos indígenas se considera el desarrollo de una educación propia, apropiada y autodeterminada de todos los sectores de la población. Al mismo tiempo, una educación que integre la población de distinto género y edad; que considere y respete las estructuras sociales y organizativas propias: como la organización por clanes o familias extendidas.

La red DESC-ARUANDA fue creada para vincular a los actores y fortalecer la voz de los pueblos indígenas, con el objetivo de: Hacer ver al mundo la realidad que enfrentan a cada día; que sus comunidades existen; que sus culturas permanecen y; sistematizar sus demandas DESC para la reconstrucción de su buen vivir comunitario. La reflexión en comunidades partió de un enfoque histórico-geográfico y de la agenda indígena, organizada en tópicos: biogeografía; territorio y territorialidades; sociedad y cultura; educación; producción; género; niñez y adolescencia; adultos mayores y casos DESC.

De esta iniciativa se comprende que la cooperación con los pueblos indígenas debe darse por: 1) La investigación transversal y sistemática de los espacios comunitarios. 2) El fortalecimiento de los procesos informativos y auto-educativos comunitarios. 3) El apoyo a las organizaciones locales. 4) La vinculación entre colectividades y actores a nivel global.

Las poblaciones indígenas de Costa Rica luchan contra el olvido de sus comunidades, incoherente con las políticas de integración impuestas a nivel nacional, caracterizadas por la falta de vías comunicantes, transportes, oportunidades económicas y productivas justas y apropiadas para sus culturas, infraestructura de salud y educación. Al mismo tiempo, luchan contra la invasión de sus tierras por finqueros y empresas; además de las 
Revista Universidad en Diálogo • Vol. 6, N. ${ }^{\circ} 2$, Julio-diciembre 2016, pp. 75-92

ISSN 2215-2849 • EISSN: 2215-4752

DOI: http://dx.doi.org/10.15359/udre.6-2.5

que presentan sobreposición de dominios, como las tierras registradas como parques nacionales, reservas forestales, entre otras. Entre los principales problemas cotidianamente enfrentados por los pueblos indígenas en Costa Rica se destacan los de la educación, la salud, la producción y el ambiente.

Entre las vías de cooperación abordadas en esta experiencia de trabajo con los pueblos indígenas de Costa Rica, se considera la responsabilidad desde la academia, en específico, de las universidades públicas de Costa Rica, en el sentido de generar espacios de construcción de un diálogo con las comunidades, el cual, desde la perspectiva humanista del CEG/UNA, se considera como un espacio democrático de fortalecimiento de su ciudadanía, mediante el fortalecimiento de sus DESC. En estos términos, la estrategia de entrada de este proyecto se alinea a la lucha por la autodeterminación, mediante el desarrollo de oportunidades de capacitación o formación, en el marco del humanismo y de la construcción de saberes interculturales.

Dos necesidades básicas se avizoran en la construcción de este proceso con los pueblos indígenas en Costa Rica: 1) La construcción de información sistemática y cercana a la realidad de las comunidades locales. 2) E1 desarrollo participativo de planes de formación indígena, en el marco de quehacer cotidiano de la universidad. Ambos factores citados, son precisamente los aspectos de mayor carencia dentro del escenario nacional, a la vez obscurecidos por los problemas organizativos locales, por la individualidad propia del trabajo académic, y por la falta de canales de articulación de los diferentes escenarios.

Sin embargo, en este momento, es importante mantener y potenciar el diálogo e interacción entre los pueblos indígenas y el mundo exterior, mediante acciones concretas, que favorezcan la sostenibilidad y el éxito del trabajo colectivo, considerando la historia de exterminio de que fueron víctimas, y que esta historia no se ha borrado de su memoria colectiva. Es fundamental favorecer la incidencia de los pueblos indígenas como un continuo de pequeñas colectividades y familias (clanes), en estadios distintos de asimilación de esta planteada -relación necesaria- con la sociedad externa. Al mismo tiempo, como colectividades que sufren los efectos profundos de la alteración de sus formas ancestrales de vida, partiendo de cambios espirituales, dados por la intervención en su vida material, resumidos en el desarrollo de actividades comerciales o extractivas de sus recursos naturales: minería, agua y potencial hidroeléctrico, y tierras cultivables industrialmente y susceptibles del pago por servicios ambientales (Borge Carbajal, 2007). 
Las intervenciones afectan la dinámica de la vida local, generando enfermedades diversas de carácter colectivo como el alcoholismo masivo, principalmente de hombres, que causa la disolución de hogares, y afecta a mujeres que pasan a administrar y proveer para toda la familia. Los demás problemas de salud, históricamente, se configuran como la contaminación de los pueblos con enfermedades de carácter urbano, antes desconocidas para ellos y para las cuales (pese los ya 500 años de conquista) siguen generando defensas, como en el caso de la propia gripe, o de la tos ferina persistente en Chirripó (López, Campos y Stoian, 2006).

Es inevitable hablar de los complejos procesos de erosión de las prácticas culturales que enfrentan los pueblos indígenas, a medida que entran en contacto con pensamientos, prácticas y tecnologías foráneas. Pero, también, por la pérdida de áreas productivas de su territorio, invadidas por plagas, o contaminadas con agroquímicos y desechos orgánicos e inorgánicos de producciones o fincas vecinas. Sin tener el dinero suficiente para resistir a las citadas adversidades, los indígenas son la población más vulnerable de Costa Rica y, visiblemente, la más afectada por los modelos de desarrollo económico impuestos, y por la negación de su existencia como culturas autodeterminadas en sus propios territorios originarios.

Si se considera que históricamente se ha visto en diferentes países, como el Ecuador con gran experiencia en el desarrollo de educación intercultural indígena, que la oportunidad educación apropiada se transforma en un medio muy importante para el reposicionamiento de los pueblos indígenas y la reconstrucción de sus culturas, esta debe llegar formando cuadro locales, los cuales tengan las potencialidades de multiplicar los conocimientos dentro de sus comunidades. De este modo, las universidades se vuelven espacios estratégicos para la formación de actores clave para que trabajen en sus comunidades. 
Revista Universidad en Diálogo • Vol. 6, N. ${ }^{\circ} 2$, Julio-diciembre 2016, pp. 75-92

ISSN 2215-2849 • EISSN: 2215-4752

DOI: http://dx.doi.org/10.15359/udre.6-2.5

\section{Referencias}

Andrés, M., Campos, J. J., Villalobos, R. y. Stoian, D. (2006). Estrategias de vida en comunidades indigenas cabécares de Alto Chirripó, Costa Rica. Incidencia en el aprovechamiento y comercialización de productos forestales y agropecuarios (Serie técnica. Informe técnico no. 346). Gestión Integrada de Recursos Naturales a Escala de Paisaje. Publicación N. ${ }^{\circ}$ 2. Costa Rica: CATIE.

Borge, C. (2007). Consulta en los territorios indígenas del Pacífico de Costa Rica. Regularización del Catastro y Registro Nacional de la Propiedad Imueble y su Compatibilización con el Registro Contrato de Préstamo BID 1284/OC-CR (Serie: Investigación y Análisis N. ${ }^{\circ}$ 2. Componente 2). San José, Costa Rica: Unidad Ejecutora Programa de Regularización de Catastro y Registro.

Bush, L. \& C. Sachs (1981). The agricultural sciences and the modern world system. In L. Buss (Ed.), Science and agricultural development. Totowa, N.J.: Alanheld, Osmu.

Ferrero, L. (2000). Costa Rica precolombina. Editorial Costa Rica. 488 p.

Fernández, (2006) Reseña Histórica de Talamanca. San José, Costa Rica: EUNED.

Fernández, R. (2008). Cartilla histórica de Costa Rica I (Hasta 1948). San José, Costa Rica: Litografía e Imprenta Lil.

González, M. (2009). Lo propio, lo nuestro, lo de Todos: Educación e interculturalidad. En M. González (Ed.). San José, Costa Rica: MEP.

Leal, D. y Vega. H. (06-10 de octubre, 2014). Comunidades autóctonas, ética y educación intercultural para la reconstrucción. Ponencia presentada en el III Congreso Internacional de Educación Superior: "Fomentar la solidaridad compartiendo el saber". Universidad Nacional, Costa Rica.

Molina, I. y Palmer, S. (2007). Historia de Costa Rica: Breve, actualizada $y$ con ilustraciones ( $2^{\mathrm{a}}$. Edición revisada). San José: EUCR.

Rojas, J. R. (2006). Así era Curré: Una visión de la comunidad indígena de Curré de principios del Siglo XIX hasta la década de los años 50 (Serie Historia Local N. ${ }^{\circ}$ 2). San José: EUNED.

Sibaja, L. F. (2006). El cuarto viaje de Cristóbal Colón y los orígenes de la provincia de Costa Rica. Colección V Centenario del IV Viaje de Cristóbal Colón N. ${ }^{\circ}$ 1. San José: EUNED. 